Research Article

Open Access

Nina Lebedeva* and Anton Petrunin

\title{
5-Point CAT(0) Spaces after Tetsu Toyoda
}

https://doi.org/10.1515/agms-2020-0126

Received September 24, 2020; accepted June 15, 2021

Abstract: We give another proof of Toyoda's theorem that describes 5-point subspaces in CAT(0) length spaces.

Keywords: $\operatorname{CAT}(0)$; finite metric space; comparison inequality; Alexandrov comparison

MSC: 53C23, 30L15, 51F99

\section{Introduction}

The CAT( 0 ) comparison is a certain inequality for 6 distances between 4 points in a metric space. The following descriptions, the so-called (2+2)-comparison, is the most standard, we refer to [3,4] for other definitions and their equivalences.

Given a quadruple of points $p, q, x, y$ in a metric space $X$, consider two model triangles (that is, a plane triangle with the same sides) $[\tilde{p} \tilde{x} \tilde{y}]=\tilde{\triangle}(p x y)$ and $[\tilde{q} \tilde{x} \tilde{y}]=\tilde{\triangle}(q x y)$ with common side $[\tilde{x} \tilde{y}]$.

If the inequality

$$
|p-q|_{X} \leqslant|\tilde{p}-\tilde{z}|+|\tilde{z}-\tilde{q}|
$$

holds for any point $\tilde{z} \in[\tilde{x} \tilde{y}]$, then we say that the quadruple $p, q, x, y$ satisfies CAT(0) comparison; here $|p-q|_{X}$ denotes the distance from $p$ to $q$ in $X$.

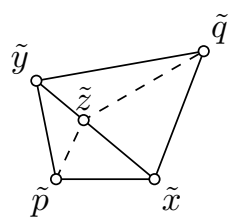

If CAT(0) comparison holds for any quadruple (and any of its relabeling) in a metric space $X$, then we say that $X$ is $\operatorname{CAT}(0)$.

It is not hard to check that if a quadruple of points satisfies CAT(0) comparison for all relabeling, then it admits a distance-preserving inclusion into a length CAT(0) space. The following theorem generalizes this statement to 5-point metric spaces.

1.1. Toyoda's theorem. Let $P$ be a 5-point metric space that satisfies CAT(0) comparison. Then $P$ admits a distance-preserving inclusion into a length $\mathrm{CAT}(0)$ space $X$.

Moreover, $X$ can be chosen to be a subcomplex of a 4-simplex such that (1) each simplex in $X$ has Euclidean metric and (2) the inclusion maps the 5 points on $P$ to the vertexes of the simplex.

A slightly weaker version of this theorem was proved by Tetsu Toyoda [7]. Our proof is shorter; it uses the fact that convex spacelike hypersurfaces in $\mathbb{R}^{3,1}$ equipped with the induced length metrics are CAT(0) spaces

${ }^{\star}$ Corresponding Author: Nina Lebedeva: Saint Petersburg State University, Saint Petersburg, Russia and Steklov Institute of Mathematics, Saint Petersburg, Russia, E-mail: lebed@pdmi.ras.ru

Anton Petrunin: Mathematics Department, Pennsylvania State University, University Park, USA, E-mail: petrunin@math.psu.edu 
[1]. We construct a distance-preserving inclusion $\iota$ of $P$ into $\mathbb{R}^{4}$ or $\mathbb{R}^{3,1}$. In the case of $\mathbb{R}^{4}$ the convex hull $K$ of $\iota(P)$ can be taken as $X$; in the case of $\mathbb{R}^{3,1}$ we take as $X$ a spacelike part of the boundary of $K$.

It is expected that any 5 point metric space $P$ as in the theorem admits a distance-preserving inclusion in a product of trees.

An analog of Toyoda's theorem does not hold for 6-point sets. It can be seen by using the so-called (4+2)comparison introduced in [2]; this comparison holds for any length CAT(0) space, but may not hold for a space with $\operatorname{CAT}(0)$ comparison (if it is not a length space).

The (4+2)-comparison is not a sufficient condition for 6-point spaces. More precisely, there are 6-point metric spaces that satisfy $(4+2)$ and $(2+2)$-comparisons but do not admit a distance-preserving embedding into a length CAT(0) space. An example was constructed by the first author; it is described in [2] right after 7.2. See the final section for related questions.

\section{5-point arrays in 3-space}

Denote by $\mathcal{A}$ the space of all 5 point arrays in $\mathbb{R}^{3}$ that is nondegenerate in the following sense: (1) all 5 points do not lie on one plane and (2) no three points lie on one line. Note that $\mathcal{A}$ is connected.

A 5 point array $x_{1}, \ldots, x_{5} \in \mathbb{R}^{3}$ defines an affine map from a 4-simplex to $\mathbb{R}^{3}$. Fix an orientation of the 4-simplex and consider the induced orientations on its 5 facets. Each facet may be mapped in an orientation-preserving, degenerate, or orientation-reversing way. For each array consider the triple of integers $\left(n_{+}, n_{0}, n_{-}\right)$, where $n_{+}, n_{0}$, and $n_{-}$denote the number of orientation-preserving, degenerate, or orientationreversing facets respectively.

Clearly $n_{+}+n_{0}+n_{-}=5$ and since all 5 points cannot lie in one plane, we have that $n_{+} \geqslant 1, n_{-} \geqslant 1$, and $n_{0} \leqslant 1$. Therefore, the value $m=n_{-}-n_{+}$can take an integer value between -3 and 3 ; in this case, we say that an array belongs to $\mathcal{A}_{m}$.

It defines a subdivision of $\mathcal{A}$ into 7 subsets $\mathcal{A}_{-3}, \ldots, \mathcal{A}_{3}$ with combinatorial configuration as on the diagram; quadruples in one plane are marked in gray and the triple $\left(n_{+}, n_{0}, n_{-}\right)$is written below.

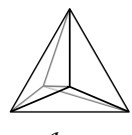

$\mathcal{A}_{-3}$

$(1,0,4)$

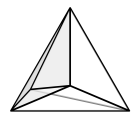

$\mathcal{A}_{-2}$

$(1,1,3)$

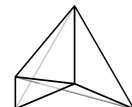

$\mathcal{A}_{-1}$

$(2,0,3)$

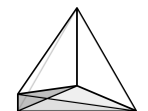

$\mathcal{A}_{0}$

$(2,1,2)$
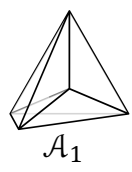

$(3,0,2)$

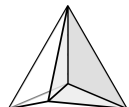

$\mathcal{A}_{2}$

$(3,1,1)$

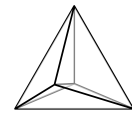

$\mathcal{A}_{3}$

$(4,0,1)$

Every two quadrilaterals in the array have 3 common points that define a plane. If the remaining two points lie on opposite sides from the plane, then the corresponding facets have the same orientation; if they lie on one side, then the orientations are opposite. Therefore, the 7 subsets $\mathcal{A}_{-3}, \ldots \mathcal{A}_{3}$ can be described in the following way:

$\mathcal{A}_{-3}-$ a tetrahedron with preserved orientation and one point inside.

$\mathcal{A}_{-2}-$ a tetrahedron with preserved orientation and one point on a facet.

$\mathcal{A}_{-1}-$ a double triangular pyramid formed by two tetrahedrons with preserved orientation.

$\mathcal{A}_{0}$ - a pyramid over a convex quadrilateral

$\mathcal{A}_{1}$ - a double triangular pyramid formed by two tetrahedrons with reversed orientation.

$\mathcal{A}_{2}-$ a tetrahedron with reversed orientation and one point on a facet.

$\mathcal{A}_{3}-\mathrm{a}$ tetrahedron with reversed orientation and one point inside.

Note that the complement $\mathcal{A} \backslash \mathcal{A}_{0}$ has two connected components formed by $\mathcal{A}_{-}=\mathcal{A}_{-3} \cup \mathcal{A}_{-2} \cup \mathcal{A}_{-1}$ and $\mathcal{A}_{+}=\mathcal{A}_{3} \cup \mathcal{A}_{2} \cup \mathcal{A}_{1}$. Observe that each array in $\mathcal{A}_{-}$has at least 3 positively oriented facets and each array in $\mathcal{A}_{+}$has at least 3 negatively oriented facets.

2.1. Observation. Let $Q$ be a connected subset of $\mathcal{A}$ that does not intersect $\mathcal{A}_{0}$. Then either $Q \subset \mathcal{A}_{+}$or $Q \subset \mathcal{A}_{-}$. 


\section{Associated form}

In this section we recall some facts about the so-called associated form introduced in [6]; it is a quadratic form $W_{\boldsymbol{X}}$ on $\mathbb{R}^{n-1}$ associated to a given $n$-point array $\boldsymbol{x}=\left(x_{1}, \ldots, x_{n}\right)$ in a metric space $X$.

Construction. Let $\triangle$ be the standard simplex $\triangle$ in $\mathbb{R}^{n-1}$; that is, the first $(n-1)$ of its vertices $v_{1}, \ldots, v_{n}$ form the standard basis on $\mathbb{R}^{n-1}$, and $v_{n}=0$.

Recall that $|a-b|_{X}$ denotes the distance between points $a$ and $b$ in the metric space $X$. Set

$$
W_{\boldsymbol{x}}\left(v_{i}-v_{j}\right)=\left|x_{i}-x_{j}\right|_{X}^{2}
$$

for all $i$ and $j$. Note that this identity defines $W_{x}$ uniquely.

The constructed quadratic form $W_{\boldsymbol{x}}$ will be called the form associated to the point array $\boldsymbol{x}$.

Note that an array $\boldsymbol{x}=\left(x_{1}, \ldots, x_{n}\right)$ in a metric space $X$ is isometric to an array in Euclidean space if and only if $W_{\boldsymbol{x}}(v) \geqslant 0$ for any $v \in \mathbb{R}^{n-1}$.

In particular, the condition $W_{\boldsymbol{x}} \geqslant 0$ for a triple $\boldsymbol{x}=\left(x_{1}, x_{2}, x_{3}\right)$ means that all three triangle inequalities for the distances between $x_{1}, x_{2}$, and $x_{3}$ hold. For an $n$-point array, it implies that $W_{\boldsymbol{x}}(v) \geqslant 0$ for any vector $v$ in a plane spanned by a triple $x_{i}, x_{j}, x_{k}$. In particular, we get the following:

3.1. Observation. Let $W_{\boldsymbol{x}}$ be a form on $\mathbb{R}^{n-1}$ associated with a point array $\boldsymbol{x}=\left(x_{1}, \ldots, x_{n}\right)$. Suppose that $L$ is a subspace of $\mathbb{R}^{n-1}$ such that $W_{\boldsymbol{x}}(v)<0$ for any nonzero vector $v \in L$. Then the projections of any 3 vertices of $\triangle$ to the quotient space $\mathbb{R}^{n-1} / L$ are not collinear.

CAT(0) condition. Consider a point array $\boldsymbol{x}$ with 4 points. From 3.1, it follows that $W_{\boldsymbol{X}}$ is nonnegative on every plane parallel to a face of the tetrahedron $\triangle$. In particular, $W_{\boldsymbol{x}}$ can have at most one negative eigenvalue.

Assume $W_{\boldsymbol{x}}(w)<0$ for some $w \in \mathbb{R}^{3}$. From 3.1, the line $L_{w}$ spanned by $w$ is transversal to each of 4 planes parallel to a face of $\triangle$.

Consider the projection of $\triangle$ along $L_{w}$ to a transversal plane. The projection of the 4 vertices of $\triangle$ lie in general position; that is, no three of them lie on one line. Therefore, we can see one of two combinatorial pictures shown on the diagram. Since the set of lines $L_{w}$ with $W_{\boldsymbol{x}}(w)<0$ is connected, the combinatorics of the picture does not depend on the choice of $w$.

3.2. Claim. If $\mathrm{CAT}(0)$ comparison holds in $X$, then the diagram on the right cannot appear.

(The converse holds as well, but we will not need it.)
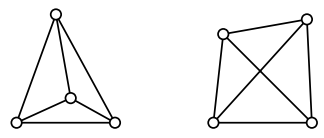

Proof. Suppose we see the picture on the right.

Let $\left[v_{1}, v_{3}\right]$ and $\left[v_{2}, v_{4}\right]$ be the line segments of $\Delta$ that correspond to the diagonals on the picture. Denote by $m$ the point of $\left[v_{1}, v_{3}\right]$ that corresponds to the point of intersection.

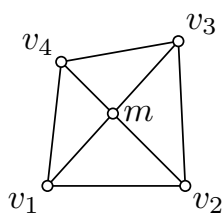

In the plane spanned by $\left[v_{2}, v_{4}\right]$ and $w$, the vector $w$ is timelike. Therefore we have the following reversed triangle inequality:

$$
\left|v_{2}-m\right|+\left|v_{4}-m\right|<\left|v_{2}-v_{4}\right| ;
$$


here we use shortcut $|a-b|=\sqrt{W(a-b)}$.

Note that the triangles $\left[v_{1} v_{2} v_{3}\right]$ and $\left[v_{1} v_{3} v_{4}\right]$ with metric induced by $W$ are isometric to model triangles of $\left[x_{1} x_{2} x_{3}\right]$ and $\left[x_{1} x_{3} x_{4}\right]$. Whence (2+2)-point comparison does not hold.

The claim implies the following:

3.3. Observation. Suppose a metric on $\boldsymbol{x}=\left(x_{1}, \ldots, x_{n}\right)$ satisfies CAT(0) comparison and $W_{\boldsymbol{x}}$ is its associated form on $\mathbb{R}^{n-1}$. Assume that $L$ is a subspace of $\mathbb{R}^{n-1}$ such that $W_{\boldsymbol{x}}(v)<0$ for any nonzero vector $v \in L$. Then if the projections of 4 vertices of $\triangle$ to the quotient space $\mathbb{R}^{n-1} / L$ lies in one plane, then its projection looks like the picture on the left; that is, one of the points lies in the triangle formed by the remaining three points.

3.4. Corollary. Suppose a metric on $\boldsymbol{x}=\left(x_{1}, \ldots, x_{5}\right)$ satisfies CAT( 0$)$ comparison and $W_{\boldsymbol{x}}$ is its associated form on $\mathbb{R}^{4}$. Assume that $L$ is a subspace of $\mathbb{R}^{4}$ such that $W_{\boldsymbol{x}}(v)<0$ for any nonzero vector $v \in L$. Then $\operatorname{dim} L \leqslant 1$.

Moreover, if $\operatorname{dim} L=1$, then the projections of the vertices of $\triangle$ to the quotient space $\mathbb{R}^{3}=\mathbb{R}^{4} / L$ belong to $\mathcal{A} \backslash \mathcal{A}_{0}$ (defined in the previous section).

Proof. If $\operatorname{dim} L \geqslant 2$, then $\operatorname{dim}\left(\mathbb{R}^{4} / L\right) \leqslant 2$. By 3.1, these 5 projections lie in a general position; that is, no three of these projections lie on one line. Therefore, $\mathbb{R}^{4} / L=2$ is the plane.

Any 5 points in a general position on the plane include 4 vertices of a convex quadrangle. The latter contradicts 3.3 .

\section{Convex spacelike surfaces}

Let $W$ be a quadratic form on $\mathbb{R}^{4}$. Suppose that $W$ has exactly one negative eigenvalue. Choose future and past cones $C^{+}$and $C^{-}$for $W$; that is, $C^{+}$and $C^{-}$are connected components of the set $\left\{v \in \mathbb{R}^{4} \mid W(v)<0\right\}$. A subset $S$ in $\mathbb{R}^{4}$ will be called spacelike if $W(x-y) \geqslant 0$ for any $x, y \in S$.

Let $K$ be a convex body in $\mathbb{R}^{4}$; denote by $\Sigma$ the surface of $K$. A point $p$ lies on the upper side of $\Sigma$ (briefly $p \in$ $\Sigma^{+}$) if there is a spacelike hyperplane in $\mathbb{R}^{4}$ that supports $\Sigma$ at $p$ from above; more precisely if the Minkowski sum $\{p\}+C^{+}$does not intersect $K$.
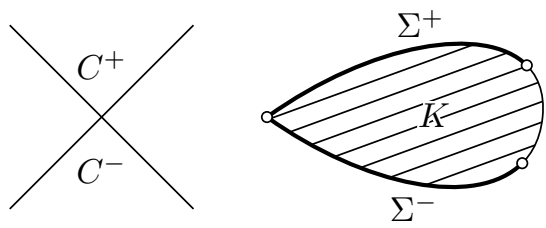

Similarly, we define the lower side of $\Sigma$ denoted by $\Sigma^{-}$. Note that $\Sigma^{+}$and $\Sigma^{-}$might have common points. The subsets $\Sigma^{+}$and $\Sigma^{-}$are spacelike; in particular, the length of any Lipschitz curve in these subsets can be defined and it leads to induced intrinsic pseudometrics on $\Sigma^{+}$and $\Sigma^{-}$. Abusing notation, we will not distinguish a pseudometric space and the corresponding metric space.

4.1. Lemma. Let $\Sigma$ be the surface of a convex set $K$ in $\mathbb{R}^{4}$ and $C^{ \pm}$be the future and past cones for a quadratic form $W$. Then the upper and lower sides $\Sigma^{+}$and $\Sigma^{-}$of $\Sigma$ equipped with the induced intrinsic metric are CAT(0) length spaces.

Moreover, if a line segment $[p q]$ in $\mathbb{R}^{4}$ lies on $\Sigma^{ \pm}$, then $[p q]$ is a minimizing geodesic in $\Sigma^{ \pm}$; that is,

$$
|p-q|_{\Sigma^{ \pm}}^{2}=W(p-q) .
$$

This lemma is essentially stated by Anatolii Milka [1, Theorem 4]; we give a sketch of alternative proof based on smooth approximation. 
Sketch. We can assume that $W$ is nondegenerate; that is, after a linear change of coordinates it is the standard form on $\mathbb{R}^{3,1}$. If not, then there is a $W$-preserving projection of $\mathbb{R}^{4}$ to a $W$-nondegenerate subspace; apply this projection and note that this subspace is isometric a subspace of $\mathbb{R}^{3,1}$.

Assume $S$ is a smooth strictly spacelike hypersurface in $\mathbb{R}^{3,1}$ with convex epigraph. By Gauss formula, $S$ has nonpositive sectional curvature.

Suppose a strictly spacelike hyperplane $\Pi$ cuts from $S$ a disc $D$. Recall that Liberman's lemma [1, Theorem 3] implies that time coordinate is convex on any geodesic in $S$. We may assume that time is vanishing on $\Pi$; therefore, by the lemma, $D$ has a convex set in $S$. Therefore the Cartan-Hadamard theorem [4] implies that that $D$ is $\operatorname{CAT}(0)$.

Now suppose $D_{n}$ is a sequence of smooth discs of the described type that converges to a (possibly nonsmooth) disc $D$. Note that the metric on $D_{n}$ converges to the induced pseudometric on $D$. It follows that the metric space $D^{\prime}$ that corresponds to $D$ is $\operatorname{CAT}(0)$.

The disc $D$ might contain lightlike segments which have zero length. Note that every maximal lightlike segment in $D$ starts at its interior point and goes to the boundary. Consider the map $\iota: D \rightarrow D$ that sends each maximal lightlike segment to its starting point. Note that the sublemma below implies that $\iota$ is lengthnonincreasing. Since $|x-\iota(x)|_{D}=0$, we get that the $D^{\prime}$ is isometric to the image of $\iota$ with the induced metric.

Consider the Minkowski sum

$$
K^{-}=K+C^{+}
$$

it has a convex spacelike boundary $\partial K^{-}$. Choose a strictly spacelike hyperplane $\Pi$ that lies above $K$. Denote by $D$ the subset of $\partial K^{-}$below $\Pi$. Let us equip $D$ with induced intrinsic pseudometric. By construction $\Sigma^{-}$is isometric to $\iota(D)$. It follows that $\Sigma^{-}$is $\operatorname{CAT}(0)$.

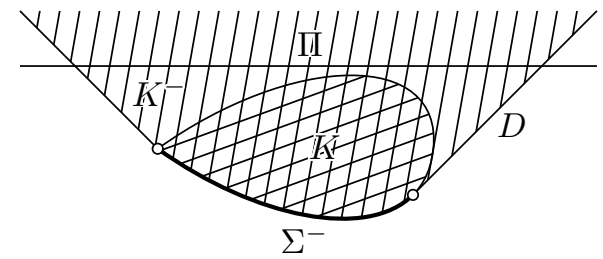

Now suppose a line segment $[p q]$ in $\mathbb{R}^{4}$ lies on $\Sigma^{-}$. Choose a supporting hyperplane $\Pi$ at the midpoint of $[p q]$. Choose time coordinate that vanish on $\Pi$; by Liberman's lemma, every shortest path in $\Sigma^{-}$between $p$ and $q$ has to lie on $\Pi$; that is, the intersection $\Sigma^{-} \cap \Pi$ is a convex subset of $\Sigma^{-}$. Therefore $[p q]$ is convex in $\Sigma^{-}$ which implies the second statement.

4.2. Sublemma. Let $u$ and $v$ be two lightlike vectors in $\mathbb{R}^{3,1}$. Suppose that the union of two half-lines $s \mapsto p+s \cdot u$ and $t \mapsto q+t \cdot v$ for $s, t \geqslant 0$ is a spacelike set. Then the function $(s, t) \mapsto|(p+s \cdot u)-(q+t \cdot v)|$ is nondecreasing in both arguments, where $|w|:=\sqrt{\langle w, w\rangle}$ for a spacelike vector $w$.

Proof. Since $u$ and $v$ are lightlike, $\langle u, u\rangle=\langle v, v\rangle=0$. Since the union of two half-lines is spacelike, $(p+s \cdot u)-(q+t \cdot v)$ is spacelike for any $s, t \geqslant 0$. It follows that

$$
\begin{aligned}
0 & \leqslant|(p+s \cdot u)-(q+t \cdot v)|^{2}= \\
& =|p-q|^{2}-2 \cdot s \cdot\langle u, q-p\rangle-2 \cdot t \cdot\langle v, p-q\rangle-2 \cdot s \cdot t \cdot\langle u, v\rangle
\end{aligned}
$$

for any $s, t \geqslant 0$. Therefore

$$
\langle u, q-p\rangle \leqslant 0, \quad\langle v, p-q\rangle \leqslant 0 \quad\langle u, v\rangle \leqslant 0 .
$$

Whence the result.

Assume $v$ is a nonzero vector in $\mathbb{R}^{4}$ and $p \in \Sigma$. We say that $p$ lies on the upper side of $\Sigma$ with respect to $v$ (briefly $p \in \Sigma^{+}(v)$ ) if $p+t \cdot v \notin K$ for any $t>0$. Correspondingly, $p$ lies on the lower side of $\Sigma$ with respect to $v$ (briefly $p \in \Sigma^{-}(v)$ ) if $p+t \cdot v \notin K$ for any $t<0$. 
4.3. Observation. Let $K$ be a compact convex set in $\mathbb{R}^{4}$ and $C^{ \pm}$be the future and past cones for a quadratic form $W$. Then the upper (lower) side of the boundary surface $\Sigma$ of $K$ can be described as the intersection of the upper (respectively lower) sides of $\Sigma$ with respect to all vectors $v \in C^{+}$; that is,

$$
\Sigma^{ \pm}=\bigcap_{v \in C^{+}} \Sigma^{ \pm}(v)
$$

\section{Proof assembling}

Proof of Toyoda's theorem. Let $\left\{x_{1}, \ldots, x_{5}\right\}$ be the points in $P$. Choose a 5-simplex $\triangle$ in $\mathbb{R}^{4}$; denote by $W$ the form associated with the point array $\left(x_{1}, \ldots, x_{5}\right)$.

If $W \geqslant 0$, then $P$ admits a distance preserving embedding into Euclidean 4-space, so one can take the convex hull of its image as $X$.

Suppose $W(v)<0$ for some $v \in \mathbb{R}^{4}$. Since $P$ is $\operatorname{CAT}(0), 3.4$ implies that $W$ has exactly one negative eigenvalue. Moreover, if a line $L$ is spanned by a vector $v$ such that $W(v)<0$, then the projection of the vertices of the simplex to $\mathbb{R}^{3}=\mathbb{R}^{4} / L$ belongs to $\mathcal{A} \backslash \mathcal{A}_{0}$.

The space of such lines $L$ is connected. By 2.1, we can assume that all the projections belong to $\mathcal{A}_{-}$. That is, we can choose timelike orientation such that for any $v \in C^{+}$the lower part $\Sigma^{-}(v)$ of $\Sigma=\partial \triangle$ has at least 3 facets of $\triangle$.

In particular, $\Sigma^{-}(v)$ contains all edges of $\triangle$ for any $v \in C^{+}$. By 4.3, $\Sigma^{-}$contains all edges of $\triangle$. By 4.1, $\Sigma^{-}$ with induced (pseudo)metric is a length $\operatorname{CAT}(0)$ space.

Since all edges of $\triangle$ lie in $\Sigma^{-}$, the inclusion $P \hookrightarrow \Sigma^{-}$is distance preserving. Whence we can take $X=\Sigma^{-}$.

Finally, observe that in each case $X$ is a subcomplex of $\triangle$ that includes all edges and has a model metric on each simplex.

\section{Remarks}

Let us recall the definition of graph comparison given by Vladimir Zolotov and the authors [5] and use it to formulate a few related questions.

Let $\Gamma$ be a graph with vertices $v_{1}, \ldots, v_{n}$. A metric space $X$ is said to meet the $\Gamma$-comparison if for any set of points in $X$ labeled by vertices of $\Gamma$ there is a model configuration $\tilde{v}_{1}, \ldots, \tilde{v}_{n}$ in the Hilbert space $\mathbb{H}$ such that if $v_{j}$ is adjacent to $v_{j}$, then

$$
\left|\tilde{v}_{i}-\tilde{v}_{j}\right|_{\mathbb{H}} \leqslant\left|v_{i}-v_{j}\right|_{X}
$$

and if $v_{j}$ is nonadjacent to $v_{j}$, then

$$
\left|\tilde{v}_{i}-\tilde{v}_{j}\right|_{\mathbb{H}} \geqslant\left|v_{i}-v_{j}\right|_{X} .
$$

The $C_{4}$-comparison (for the 4-cycle $C_{4}$ on the diagram) defines CAT(0) comparison. Tetsu Toyoda have shown that $C_{4}$-comparison imlies graph comparisons for all cycles $C_{n}$ [8]; remakably, the metric space is not assumed to be intrinsic. The $\mathrm{O}_{3}$-comparison (for the octahedron graph $\mathrm{O}_{3}$ on the diagram) defines another

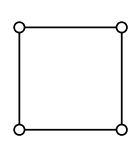

$C_{4}$

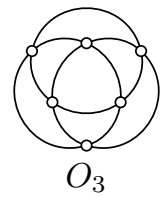

$\mathrm{O}_{3}$

comparison. Since $O_{3}$ contains $C_{4}$ as an induced subgraph, we get that $O_{3}$-comparison is stronger than $C_{4}$ comparison. 
6.1. Open question. Is it true that octahedron-comparison holds in any 6 points in a length $\mathrm{CAT}(0)$ space?

And, assuming the answer is affirmative, what about the converse: is it true that any 6-point metric space that satisfies octahedron-comparison admits a distance preserving embedding in a length $\mathrm{CAT}(0)$ space?

The analogous questions for spaces with nonnegative curvature in the sense of Alexandrov (briefly $\mathrm{CBB}(0)$ ) are open as well. The $\mathrm{CBB}(0)$ comparison is equivalent to the 3-tree comparison (for the tripod-tree shown first on the following diagram). It turns out that any length $\operatorname{CBB}(0)$ space satisfies the comparison for<smiles>O=C(O)O</smiles>

3 -tree

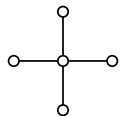

4-tree

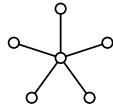

5 -tree

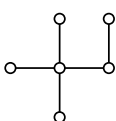

$3(1)$-tree

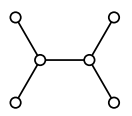

$2(2)$-tree

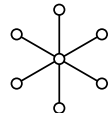

6 -tree

the other trees on the diagram; it is formed by an infinite family of star-shaped trees and two trees with 6 vertices $[2,5]$. (The 4-tree comparison (the second tree on the diagram) is equivalent to the so-called (4+1)-point comparison in the terminology of [2].)

We expect that this comparison provides a necessary and sufficient condition for 5-point sets. Namely, we expect an affirmative answer to the following stronger question.

6.2. Question. Suppose a 5-point metric space $P$ satisfies the 4-tree comparison. Is it true that $P$ admits a distance preserving embedding into a length $\mathrm{CBB}(0)$ space?

Finally, let us mention a related question about a 6-point condition.

6.3. Question. Suppose a 6-point metric space P satisfies the 5-tree, 2(2)-tree, and 3(1)-tree comparisons. Is it true that $P$ admits a distance preserving embedding into a length $\mathrm{CBB}(0)$ space?

Acknowledgment: We want to thank Stephanie Alexander, Yuri Burago, and the anonymous referee for help.

The first author was partially supported by RFBR grant 20-01-00070, the second author was partially supported by NSF grant DMS-2005279.

Conflict of interest: Authors state no conflict of interest.

\section{References}

[1] А. Д. Милка. Выпуклые гиперповерхности в псевдоевклидовом пространстве. Докл. АН СССР, 284(6):1314-1316, 1985.

[2] S. Alexander, V. Kapovitch, and A. Petrunin. Alexandrov meets Kirszbraun. In Proceedings of the Gökova Geometry-Topology Conference 2010, pages 88-109. Int. Press, Somerville, MA, 2011.

[3] S. Alexander, V. Kapovitch, and A. Petrunin. An invitation to Alexandrov geometry. SpringerBriefs in Mathematics. CAT(0) spaces.

[4] S. Alexander, V. Kapovitch, and A. Petrunin. Alexandrov geometry: preliminary version no. 1, 2019.

[5] N. Lebedeva, A. Petrunin, and V. Zolotov. Bipolar comparison. Geom. Funct. Anal., 29(1):258-282, 2019.

[6] A. Petrunin. In search of a five-point alexandrov type condition. St. Petersburg Math. J., 29(1):223-225, 2018.

[7] T. Toyoda. An intrinsic characterization of five points in a CAT(0) space. Anal. Geom. Metr. Spaces, 8(1):114-165, 2020.

[8] T. Toyoda. A non-geodesic analogue of reshetnyak's majorization theorem, 2020. 